ESJ Natural/Life/Medical Sciences

\title{
Evaluation de Différents Types de Substrats sur Le Développement des Plantules de Palmiers À Huile (Elaeis Guineensis Jacq.) en Côte d'Ivoire
}

\section{N'Guessan Assiénin Hauverset}

Centre National de Recherche Agronomique, Laboratoire d'Entomologie, Station de Recherches de La Mé, Abidjan, Côte d'Ivoire

\section{Gogoue Dessan Obed}

Centre National de Recherche Agronomique, Laboratoire d'AgronomiePhysiologie, Station de Recherches de La Mé, Abidjan, Côte d’Ivoire

\section{Anougba Bossoma Danielle}

Centre National de Recherche Agronomique, Laboratoire d'Entomologie, Station de Recherches de La Mé, Abidjan, Côte d'Ivoire

Université Nangui Abrogoua, Laboratoire d’Ecologie et Développement Durable des Ecosystèmes, Abidjan, Côte d'Ivoire

\section{Dembélé Inza}

Centre National de Recherche Agronomique, Service Exploitation, Station de Recherches de La Mé, Abidjan, Côte d’Ivoire

\section{Allou Kouassi}

Centre National de Recherche Agronomique Laboratoire d'Entomologie, Station de Recherche sur le cocotier, Marc Delorme, Port-Bouët, Abidjan,

Côte d'Ivoire

Doi:10.19044/esj.2021.v17n37p1

Submitted: 18 August 2021

Accepted: 20 September 2021

Published: 31 October 2021
Copyright 2021 Author(s)

Under Creative Commons BY-NC-ND 4.0 OPEN ACCESS

Cite As:

N’Guessan A.H., Gogoue D.O., Anougba B.D., Dembélé I. \& Allou K. (2021). Evaluation de Différents Types de Substrats sur Le Développement des Plantules de Palmiers À Huile (Elaeis Guineensis Jacq.) en Côte d'Ivoire. European Scientific Journal, ESJ, 17(37), 1. https://doi.org/10.19044/esj.2021.v17n37p1

\section{Résumé}

La Côte d'Ivoire, est le principal exportateur africain d'huile de palme au niveau de la CEDEAO. Pour maintenir sa production, de nombreuses pratiques telles que l'apport de fertilisants minéraux et organiques sont nécessaires. L’objectif général de cette étude était de comparer le développement des plantules de palmier à huile sur cinq (5) substrats 
différents constitués du terreau, de la bouse de vache et de fibres de palmier en décomposition. Les résultats sur le dénombrement de plantules mortes ont montré un faible taux de mortalité au niveau du substrat constitué uniquement du terreau $100 \%$. L'évaluation de certains paramètres morphologiques a révélé une meilleure croissance des feuilles en longueur, un nombre important de feuilles, un meilleur poids frais, un gros diamètre et une meilleure couleur verte des feuilles riches en chlorophylle chez les plantules évoluées sur les substrats de $50 \%$ de terreau, $25 \%$ de Bouse de vache et $25 \%$ de fibre de palmier, à l'exception des $100 \%$ de terreau. Il serait bon de constituer des substrats à base de bouse de vache et de fibre de palmier pour un meilleur développement du palmier à huile en pré-pépinière.

Mots clés: Taux De Mortalité, Palmier À Huile, Pré-Pépinière, Substrat, Côte d'Ivoire

\section{Evaluation of Different Types of Substrates on The Development of Oil Palm (Elaeis Guineensis Jacq.) Plantlets in Côte d'Ivoire}

\section{N'Guessan Assiénin Hauverset}

Centre National de Recherche Agronomique, Laboratoire d'Entomologie, Station de Recherches de La Mé, Abidjan, Côte d'Ivoire

\section{Gogoue Dessan Obed}

Centre National de Recherche Agronomique, Laboratoire d'AgronomiePhysiologie, Station de Recherches de La Mé, Abidjan, Côte d'Ivoire

\section{Anougba Bossoma Danielle}

Centre National de Recherche Agronomique, Laboratoire d'Entomologie, Station de Recherches de La Mé, Abidjan, Côte d'Ivoire

Université Nangui Abrogoua, Laboratoire d'Ecologie et Développement Durable des Ecosystèmes, Abidjan, Côte d'Ivoire

\section{Dembélé Inza}

Centre National de Recherche Agronomique, Service Exploitation, Station de Recherches de La Mé, Abidjan, Côte d’Ivoire

\section{Allou Kouassi}

Centre National de Recherche Agronomique Laboratoire d'Entomologie, Station de Recherche sur le cocotier, Marc Delorme, Port-Bouët, Abidjan,

Côte d'Ivoire

\section{Abstract}

Côte d'Ivoire is the main African exporter of palm oil in ECOWAS. In order to maintain its production, many practices such as the use of mineral and 
organic fertilizers are necessary. The general objective of this study was to compare the development of oil palm seedlings on five (5) different substrates consisting of potting soil, cow dung, and decomposing palm fibers. The results on the count of dead seedlings showed a low mortality rate on the $100 \%$ potting soil substrate. Evaluation of some morphological parameters revealed better leaf growth in length, a large number of leaves, better fresh weight, larger diameter and better green color of the chlorophyll-rich leaves in the evolved seedlings on the substrates of $50 \%$ potting soil, $25 \%$ cow dung, and $25 \%$ palm fiber, except for the $100 \%$ potting soil. It would be advisable to use cow dung and palm fiber substrates for better development of the oil palm in the pre-nursery.

Keywords: Mortality Rate, Oil Palm, Pre-Nursery, Substrate, Côte d’Ivoire

\section{Introduction}

Le palmier à huile représente actuellement la plante oléagineuse qui a le meilleur rendement en huile à l'hectare (Rival et Levang, 2013). En effet, il assure $39 \%$ de la production mondiale en huile végétale sur une fraction de terre agricole bien plus faible que celle consacrée aux autres plantes oléagineuses (Rival et Levang, 2013, LeCerfet al., 2016). La filière palmier à huile génère 200000 emplois réguliers faisant vivre environ 2 millions d'habitants soit 10 \% de la population ivoirienne (Palmci, 2014). La Côte d'Ivoire, produit actuellement environ 2100000 tonnes de régimes de palme à huile par an, soit 450000 tonnes d'huile de palme (Palmafrique, 2018). Ainsi, elle compte augmenter sa production 800000 tonnes d'ici 2025. La Côte d'Ivoire est le principal pays exportateur en Afrique Occidentale avec 60 \% des exportations. Elle fournit le marché régional en huiles brutes et raffinées ainsi qu'en produits dérivés (Cheynset al., 2000). Cette importante place occupée par le palmier à huile parmi les plantes oléagineuses, est due à l'utilisation de fertilisants minéraux et organiques. En effet, cultivé sur des sols tropicaux généralement très altérés, le palmier à huile a besoin de quantités assez importantes d'engrais pour atteindre de bonnes productions. Malgré l'absence d'éléments minéraux dans l'huile produite, des quantités importantes de nutriments sont mobilisées par la plante pour sa croissance végétative et sa production (Caliman, 2007). Cependant, l'utilisation excessive de composés azotés peut entraîner une pollution des eaux de surface et de profondeur, ainsi que celui de l'air. Aussi dans un contexte actuel de protection de l'environnement, il convient de promouvoir les choix des pratiques agricoles par l'utilisation des engrais verts ou engrais organiques. Selon Tougma (2006), l'apport de matière organique est très favorable à la macrofaune et microflore du sol. En effet, les engrais verts améliorent la structure et la texture du sol et stimulent l'activité microbienne dans le sol 
(Cedra, 1997). C’est dans cette optique que cette étude a été menée avec pour objectif général d'évaluer de l'effet de cinq (5) types de substrats sur le développement du palmier à huile en pré-pépinière. Spécifiquement, il s’agit d'évaluer le taux de mortalité des plantules de palmier à huile sur chaque substrat utilisé et de mesurer certains paramètres morphologiques tels que la vitesse de croissance en hauteur des plantules, le nombre de feuilles, la circonférence au collet des plants et l'aspect (couleur) des feuilles.

\section{Matériel et méthodes}

\section{Matériel végétal}

Le matériel végétal est constitué de graines germées de palmier à huile (Elaeis guineensis), de la variété Tenera de type C1001 classic (Figure 1). Cette variété est actuellement vulgarisée en Côte d'Ivoire et a une bonne production en huile (4,3 t/ha), une faible vitesse de croissance en hauteur (45 cm/an), une bonne qualité d'huile (Koutou et al., 2014, Tano et al., 2019).

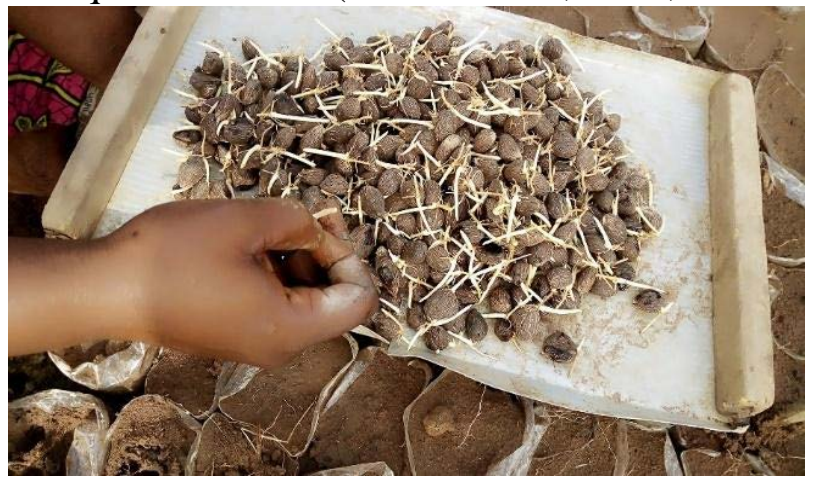

Figure 1. Graines germées de la variété C1001 de type classic

\section{Méthodologie}

\section{Site d'expérimentation}

Les travaux ont été réalisés entre le mois de mars et juin 2020, à la Station du Centre National de Recherche Agronomique (CNRA)/Côte d'Ivoire particulièrement à La Mé situé entre $5^{\circ} 26^{\prime}$ Latitude Nord et $3^{\circ} 50^{\prime}$ Longitude Ouest. Cette station est située au Sud-Est de la Côte d'Ivoire, dans le district d'Abidjan, au Nord-Est de la ville d'Abidjan à $30 \mathrm{~km}$, sur la route menant à Alépé, est bordée à l'Est par le fleuve Mé et au Sud par la lagune Aghien.

\section{Dispositif expérimental}

Le dispositif utilisé est un bloc de Fisher totalement randomisé avec 5 traitements et 4 répétitions (Figure 2). Chaque traitement constitue un bloc de 1200 graines germées. Le repiquage des graines germées a été fait après le remplissage des sachets des différents substrats. Un trou de 2 à $3 \mathrm{~cm}$ de profondeur est creusé au centre de chaque sachet au fond duquel est déposée 
la graine. Un léger arrosage a été fait après le repiquage des graines germées. Ces graines germées plantées dans des sachets, ont été suivies depuis la levée jusqu'à l'âge de 4 mois, constituant le stade pré-pépinière. Chaque bloc est subdivisé en 4 sous-blocs de 300 graines germées, reparti de façon aléatoire dans chaque répétition. Les différents traitements sont :

- T: $100 \%$ de terreau: il s'agit de la terre simple sans mélange;

- T-BV : $50 \%$ de terreau $+50 \%$ de bouse de vache avec d'un mélange d'une brouette de terreau simple et d'une brouette de bouse de vache;

- T-FP : $50 \%$ de terreau 50\% de fibres de palmier à raison d'une brouette de terreau simple et d'une brouette de fibre de palmier

- T-BV-FP : $50 \%$ de terreau $25 \%$ de bouse de vache et $25 \%$ de fibres de palmier à raison de deux brouettes de terreau simple, une brouette de bouse de vache et une brouette de fibre de palmier;

- BV-FP : 50\% de bouse de vache $50 \%$ de fibres de palmier 50 avec deux brouettes de bouse de vache et deux brouettes de fibre de palmier.

\begin{tabular}{|c|c|c|c|c|}
\hline CT & T-Bv & T-Fp & T-Bv-Fp & Bv-Fp \\
\hline Bv-Fp & $\mathrm{T}$ & $\mathrm{T}-\mathrm{Bv}$ & $\mathrm{T}-\mathrm{Fp}$ & $\mathrm{T}-\mathrm{Bv}-\mathrm{Fp}$ \\
\hline $\mathrm{T}-\mathrm{Bv}-\mathrm{Fp}$ & $\mathrm{Bv}-\mathrm{Fp}$ & $\mathrm{T}$ & $\mathrm{T}-\mathrm{Bv}$ & $\mathrm{T}-\mathrm{Fp}$ \\
\hline T-Fp & $\mathrm{T}-\mathrm{Bv}-\mathrm{Fp}$ & $\mathrm{Bv}-\mathrm{Fp}$ & $\mathrm{T}$ & $\mathrm{T}-\mathrm{Bv}$ \\
\hline
\end{tabular}

Figure 2. Dispositif expérimental en bloc de Fisher totalement randomisé

T: Terreau ; Bv: Bouse de vache; Fp: Fibres de palmier

\section{Paramètres étudiés}

Après la levée, dans chaque bloc et dans chaque sous-bloc, un dénombrement mensuel des plantules a été fait afin de relever le nombre de plantules mortes ; ce qui a permis d'évaluer le taux de mortalité au niveau de chaque substrat. Trois mois après la levée, quelques paramètres morphologiques tels que longueur des plantules, la circonférence au collet, le nombre des feuilles, l'aspect des feuilles (coloration et la biomasse) ont été mesurés. Pour ce qui concerne, la coloration des feuilles des plantules, un score de 1 à 4 a été attribué comme suit :

- 1: vert pure

- 2: vert clair

- 3: vert pale

- 4: jaunâtre 
Concernant la biomasse, 30 plantules dans chaque substrat ont été prélevées par répétition puis pesées à l'aide d'une balance de précision $0,001 \mathrm{~g}$ et de marque Adventurer.

\section{Analyse des données}

Toutes les données ont été soumises à une analyse de variance à l'aide de la procédure Général Linear Model du logiciel SAS 9.4. La comparaison des moyennes a été réalisée avec le test de LSD (Low Significant Difference) au seuil $\alpha$ de $5 \%$.

\section{Résultats}

\section{Taux de mortalité des plantules}

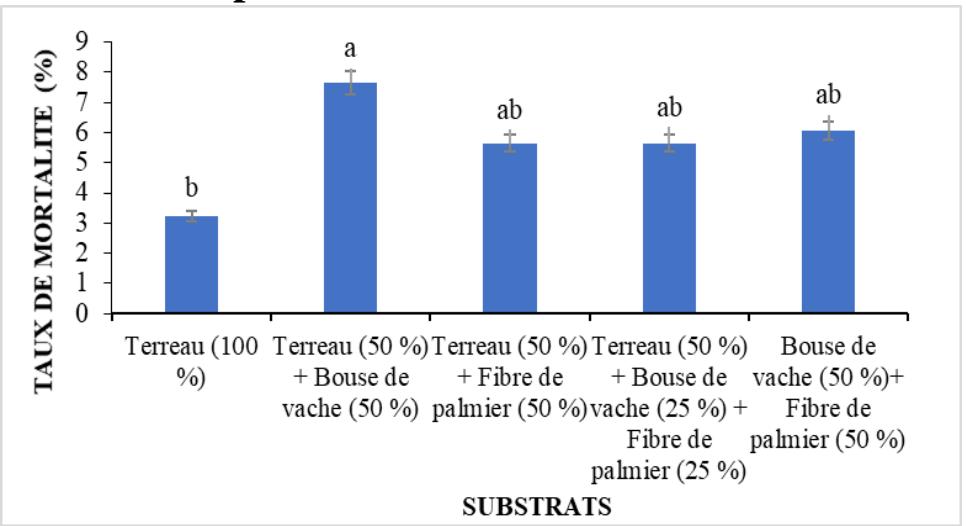

Figure 3. Taux de mortalité des plantules de palmier à huile en fonction des substrats Les barres surmontées des mêmes lettres ne sont pas très différentes au seuil $\alpha$ de $5 \%$ (LSD)

Le taux de mortalité de plantules sur le substrat composé de $50 \%$ de terreau $+50 \%$ de bouse de vache a été de 7,65 $\pm 2,94 \%$. Ces taux de mortalité ont varié selon les différents substrats utilisés en allant de 5,65 $\pm 3,73 \%$ pour $50 \%$ de terreau $+50 \%$ de fibres de palmier à $6,05 \pm 3,53 \%$ pour $50 \%$ de terreau $+25 \%$ de bouse de vache et $25 \%$ de fibre de palmier et $50 \%$ de Bouse de vache $+50 \%$ de fibre de palmier. Cependant, sur les $100 \%$ de terreau, le taux de mortalité a été de 3,24 $\pm 0,49 \%$ (Figure 3). L'analyse des variances a montré des différences significatives $(p=0,046<0,05)$ entre les substrats au regard des taux de mortalité des plantules de palmier à huile.

\section{Longueur des plantules}

La mensuration de la longueur moyenne des plantules sur le substrat composé de $50 \%$ de terreau $+25 \%$ de bouse de vache $+25 \%$ de fibres de palmier a été de 29,90 $\pm 3,69 \mathrm{~cm}$. Les longueurs de plantules observées respectivement sur les $50 \%$ de bouse de vache $+50 \%$ de fibres de palmier, les $50 \%$ de terreau $+50 \%$ de bouse de vache et les $50 \%$ de terreau $+50 \%$ 
de fibres de palmier ont été de 27,17 $\pm 4,91 \mathrm{~cm}, 26,70 \pm 4,86 \mathrm{~cm}$ et 25,76 \pm $3,95 \mathrm{~cm}$. Cependant la longueur moyenne de plantules observée avec les 100 $\%$ de terreau a été de 19,13 $\pm 4,12 \mathrm{~cm}$. Les longueurs moyennes des plantules sont statistiquement différentes $(\mathrm{P}=0,0001<0,05)$ au regard des différents substrats (Figure 4).

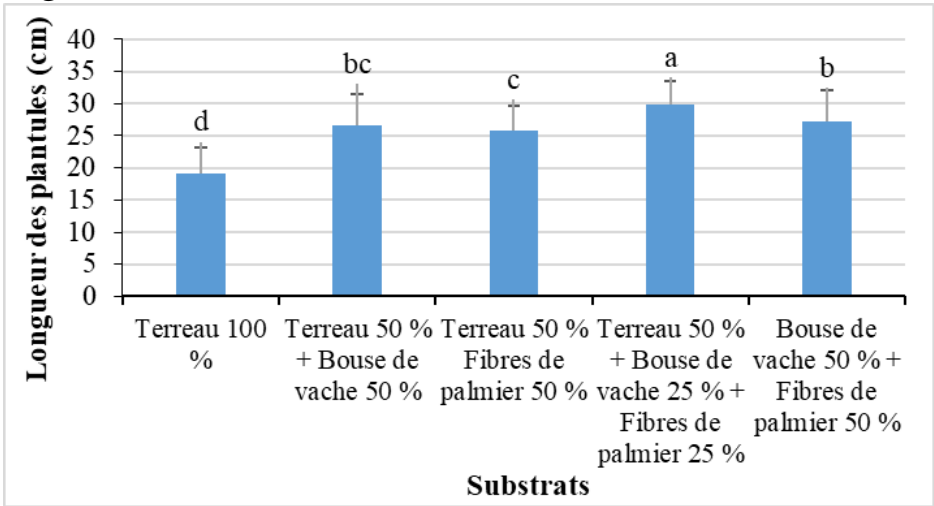

Figure 4. Mesure de la longueur des plantules

Les barres surmontées des mêmes lettres ne sont pas très différentes au seuil $\alpha$ de 5 $\%$ (LSD)

\section{Nombre de feuilles des plantules}

Les résultats du dénombrement des feuilles des plantules de palmier à huile ont montré qu'au niveau du substrat $50 \%$ de terreau $+25 \%$ de bouse de vache $+25 \%$ de fibres de palmier, le nombre moyen de feuilles a été de 4,87 $\pm 0,56$ feuilles. Le nombre moyen de feuilles obtenu avec les $50 \%$ de terreau $+50 \%$ de bouse de vache, $50 \%$ de terreau $+50 \%$ de fibres de palmier et 50 $\%$ de bouse de vache $+50 \%$ de fibres de palmier est comprise entre 4,49 \pm 0,63 feuilles et 4,63 $\pm 0,80$ feuilles. Avec les $100 \%$ de terreau, le nombre moyen de feuilles a été de 3,53 $\pm 0,66$ feuilles. Ainsi, l'analyse de variance a montré des différences significatives $(\mathrm{P}=0,0001<0,05)$ entre le nombre moyen de feuilles au regard des différents substrats (Figure 5).

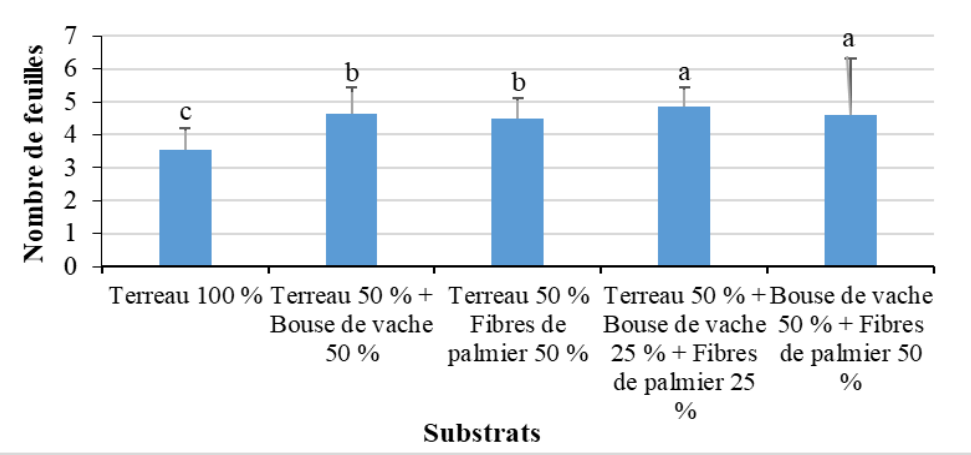

Figure 5. Nombre moyen de feuilles des plantules en fonction des substrats

Les barres surmontées des mêmes lettres ne sont pas très différentes au seuil $\alpha$ de $5 \%$ (LSD) 


\section{Couleur des feuilles des plantules}

L'analyse de la couleur des feuilles a montré que les plantules possédant les feuilles jaunâtres ont été répertoriées sur les $100 \%$ de terreau avec une moyenne de $4 \pm 00$. Les feuilles des plantules sur les $50 \%$ de terreau $+50 \%$ de fibres de palmier sont de couleur vert clair avec une moyenne de $2,5 \pm 0,59$. La couleur vert pure a été observée sur les plantules évoluant respectivement sur $50 \%$ de terreau $+25 \%$ de bouse de vache $+25 \%$ de fibres de palmier et $50 \%$ de Bouse de vache $+50 \%$ de fibres de palmier avec des valeurs comprises entre $1 \pm 00$ et $1,25 \pm 0,5$. Ainsi, l'analyse de variance a montré des différences significatives $(\mathrm{P}=0,0001<0,05)$ entre les substrats au regard de la couleur des feuilles des plantules (Figure 6 ).

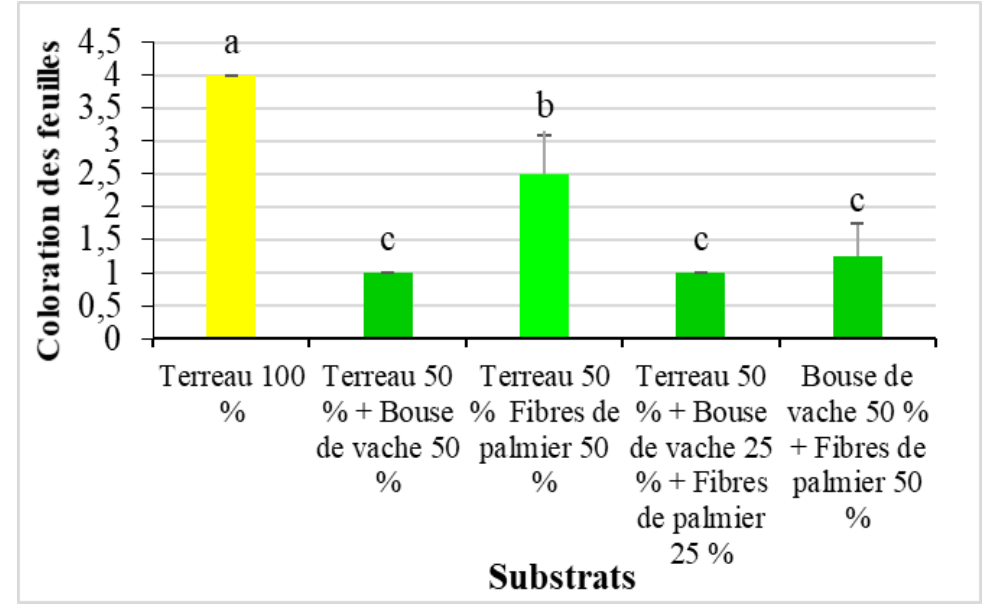

Figure 6. Observations sur la coloration des feuilles des plantules en fonction des substrats Les barres surmontées des mêmes lettres ne sont pas très différentes au seuil $\alpha$ de $5 \%$ (LSD)

\section{Diamètre au collet}

Les mesures du diamètre au collet des plantules ont donné des valeurs respectives de $8,57 \pm 1,71 \mathrm{~cm}$ au niveau des substrats composés de $50 \%$ de bouse de vache $+50 \%$ de fibres de palmier, 8,62 $\pm 1,32 \mathrm{~cm}$ au niveau des 50 $\%$ de terreau $+25 \%$ de bouse de vache $+25 \%$ de fibres de palmier et 8,66 \pm $1,40 \mathrm{~cm}$ pour $50 \%$ de terreau $+25 \%$ de bouse de vache. La mesure du diamètre au collet a été de $8,12 \pm 1,35 \mathrm{~cm}$ au niveau des $50 \%$ de terreau +50 $\%$ de fibres de palmier. Cependant avec le Terreau simple (100 \%), le diamètre au collet des plantules a été de 6,11 $\pm 1,14 \mathrm{~cm}$. Toutes ces valeurs sont statistiquement différentes $(\mathrm{P}=0,0001<0,05)$ (Figure 7). 


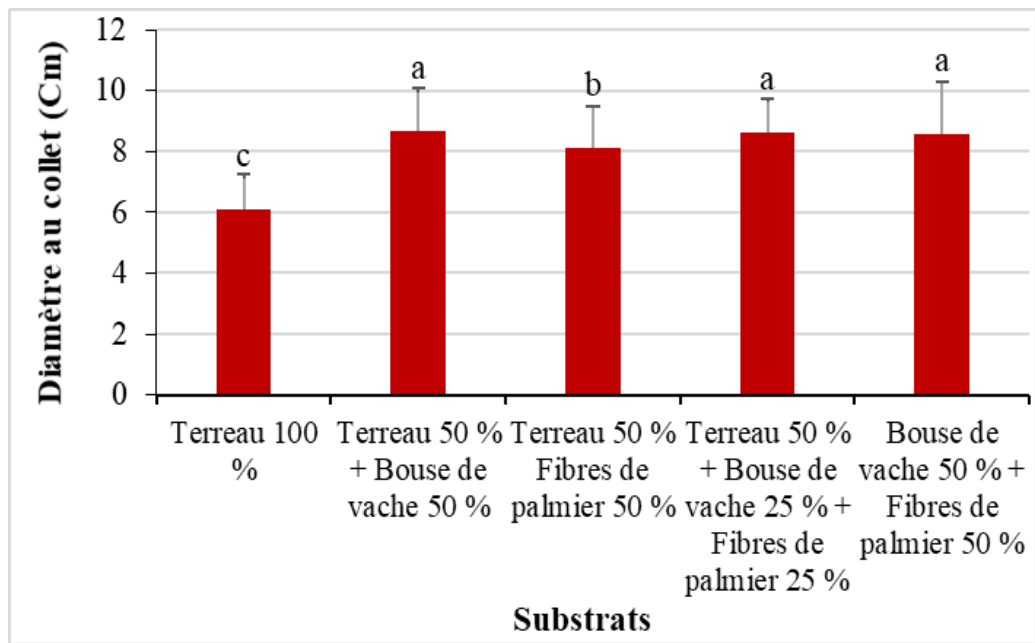

Figure 7. Mesure du diamètre au collet des plantules

Les barres surmontées des mêmes lettres ne sont pas très différentes au seuil $\alpha$ de $5 \%$ (LSD)

Biomasse fraiche

Les résultats obtenus quant à la biomasse fraiche ont révélé un poids frais moyen de 16,86 $\pm 3,56$ g avec le substrat composé de $50 \%$ de terreau + $25 \%$ de bouse de vache $+25 \%$ de fibres de palmier. Le poids frais moyen obtenu avec le substrat composé de $50 \%$ de bouse de vache $+50 \%$ de fibres de palmier a été de 14,69 $\pm 3,42 \mathrm{~g}$. Le poids frais moyen obtenu avec les $50 \%$ de terreau $+50 \%$ de bouse de vache et les $50 \%$ de terreau $+50 \%$ de fibres de palmier a été respectivement de $14,25 \pm 4,32 \mathrm{~g}$ et $13,41 \pm 3,09 \mathrm{~g}$. Cependant, un poids frais moyen de 7,26 $\pm 2,18 \mathrm{~g}$ a été obtenu avec les $100 \%$ de terreau. Ces résultats sont statistiquement différents $(\mathrm{P}=0,0001<0,05)$ (Figure 8).

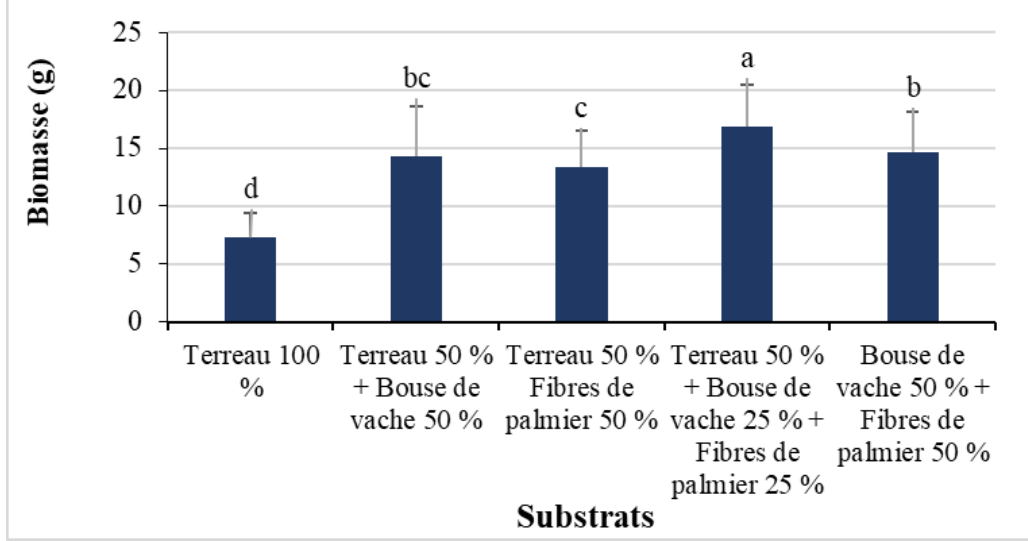

Figure 8. Mesures de la biomasse fraiche des plantules en fonction des substrats Les barres surmontées des mêmes lettres ne sont pas très différentes au seuil $\alpha$ de 5 $\%$ (LSD) 


\section{Discussion}

Une bonne production du palmier à huile implique un développement de la plante. De ce fait, la fertilité des sols sous palmiers signifie qu'un apport conséquent de nutriments minéraux ou organiques a été réalisé, ce qui a permet d'améliorer la structure de ces sols. Selon (Cedra, 1997), les engrais verts améliorent la structure du sol et stimulent l'activité microbienne dans le sol. Cette étude basée sur l'utilisation de différents substrats pour comparer le développement de plantules de palmiers à huile en pré-pépinière, a révélé un taux de mortalité élevé des plantules sur le substrat composé de $50 \%$ de terreau $+50 \%$ de Bouse de vache. Ce fort taux de mortalité observé sur ce substrat pourrait s'expliquer par le fait que ce substrat serait contaminé par certains organismes phytopathogènes, entrainant la mort d'un nombre élevé de plantules. Selon Jean-Damien (2004), les Nématodes, Enchytrées, Lombrics, Acariens, Collemboles et la microflore (bactéries, champignons et protozoaires) indispensable dans le processus de minéralisation, constituent le reste de la faune des bouses. En effet, certains de ces organismes seraient nuisibles aux plantules de palmiers à huile. Cependant, un faible taux de mortalité a été observé sur les 100 \% de Terreau. Cela serait dû à un bon choix du terreau. Le terreau de forêt prélevé dans les 10 premiers centimètres de sol, de préférence léger et humifère est bon pour la pré-pépinière de palmier à huile (Jacquemard, 2013).

Au cours de cette étude, certains paramètres morphologiques des plantules ont été mesurés. La mensuration de la longueur des plantules a montré que sur les substrats $50 \%$ de terreau $+25 \%$ de bouse de vache +25 $\%$ de fibres de palmier et $50 \%$ de bouse de vache $+50 \%$ de fibres de palmier, les longueurs des plantules sont plus élevées. En effet, ces substrats contiendraient suffisamment de nutriments, nécessaires à un développement rapide des plantules. La bouse de vache contient des teneurs élevées en phosphore total, phosphore soluble dans l'eau et azote total (Beenaert et Bitongo, 2004), en plus des autres constituants organiques contenus dans le terreau et les fibres de palmiers. Ce qui aurait permis une croissance rapide des plantules. Par contre pour les faibles longueurs de plantules sur les $100 \%$ de Terreau, il y aurait une insuffisance de matières organiques et minérales dans le terreau simple. Ce qui a eu pour conséquence le ralentissement de la croissance des plantules sur ce substrat.

L’évaluation du nombre de feuilles a donné un nombre plus élevé de feuilles sur tous les substrats excepté le terreau simple sur lequel un faible nombre a été observé. Ce résultat corrobore ceux de Ognalaga et al. (2017) qui ont indiqué que la combinaison de la fumure organique et minérale améliore significativement de la croissance, la production et le rendement de la variété du manioc, expliquant ainsi le nombre élevé de feuilles sur les substrats composés de $50 \%$ de terreau $+25 \%$ de bouse de vache $+25 \%$ de fibres de 
palmier, $50 \%$ de terreau $+50 \%$ de bouse de vache, $50 \%$ de terreau $+50 \%$ de fibres de palmier, et $50 \%$ de Bouse de vache $+50 \%$ de fibres de palmier par rapport aux $100 \%$ de terreau dans l'étude.

Les résultats des mesures du diamètre au collet des plantules ont révélé que les plantules observées sur les $50 \%$ de terreau $+50 \%$ de bouse de vache, $50 \%$ de terreau $+25 \%$ de bouse de vache $+25 \%$ de Fibres de palmier, $50 \%$ de bouse de vache $+50 \%$ de fibres de palmier ont eu une circonférence élevée par rapport autres substrats. Selon les travaux de Bakayoko et al. (2019), l'association de la bouse de vache et les autres constituants organiques entraîne une augmentation de la litière organique, consécutive à une amélioration de la fertilité du sol au niveau de ces substrats. En effet, les engrais organiques permettent au sol d'avoir une grande capacité de rétention des éléments nutritifs et de l'eau, et une grande capacité d'échange cationique, entraînant ainsi une augmentation des diamètres au collet des plantules.

Au niveau de la biomasse, les résultats obtenus montrent que les $50 \%$ de terreau $+25 \%$ de bouse de vache $+25 \%$ de fibres de palmier et $50 \%$ de bouse de vache $+50 \%$ de fibres de palmier ont donné des poids frais supérieurs autres substrats. Cela pourrait être dû à une bonne nutrition minérale et organique des plantules. La viabilité d'une terre dépend avant tout de sa richesse en humus (Tognetti et al., 2008), ce qui fait de l'utilisation des déjections animales une pratique courante en agriculture et constitue une valorisation des sous-produits de l'élevage fortement vulgarisés par l'agriculture biologique. Aussi, les fibres de palmier obtenues à partir des stipes en décomposition et les rafles, riches en matière organique et éléments fertilisants servent de compostage dans la plantation (CNUCED, 2016). Tous ces éléments auraient servi à enrichir le sol et nourrir les plantules, donc à augmenter leur poids.

Quant à la couleur des feuilles des plantules, la couleur verte a été observée sur tous les substrats à l'exception des $100 \%$ de terreau où les feuilles étaient de couleur jaunâtre. Cette couleur jaunâtre des feuilles sur terreau simple peut être due à l'absence de certains éléments minéraux ou organiques dans le sol dont les plantules ont besoin pour leur croissance. Ce qui aurait eu pour conséquence le faible taux de chlorophylles dans les plantules sur le terreau simple. Guilbault (2003) et Jacquemard (2011) stipulaient que la déficience azotée se caractérise par une teinte vert-jaune à jaune des jeunes feuilles de palmier. En outre, Jacquemard (2011) a indiqué que les carences en soufre sont rares mais peuvent apparaître sur des sols ferralitiques très désaturés, surtout sur des arbres jeunes. Alors que le site d'étude, la station de recherche de la Mé est composée de sols désaturés (Kabrah et Ballo, 2000) à l'origine de la couleur jaunâtre des plantules sur le substrat composé de $100 \%$ de terreau 


\section{Conclusion}

Les résultats obtenus lors de cette étude ont montré que les plus forts taux de mortalité ont été constatés sur les substrats comportant la bouse de vache probablement du fait la présence de certains organismes phytopathogènes tandis que les plus faibles taux ont été observés les 100 \% de terreau. Par ailleurs, l'évaluation des paramètres morphologiques a révélé une longueur des feuilles élevée, un nombre important de feuilles, un poids frais élevé, une circonférence élevée et une bonne quantité de chlorophylle (couleur verte) chez les plantules évoluant sur tous les substrats surtout sur le substrat composé de $50 \%$ de terreau $+25 \%$ de bouse de vache $+25 \%$ de fibres de palmier, à l'exception du terreau $100 \%$. Il serait bon de constituer des substrats à base de bouse de vache et de fibres de palmier pour un meilleur développement du palmier à huile en pré-pépinière.

\section{Remerciements}

Les remerciements vont à l'endroit du service Exploitation du Centre National de Recherche Agronomique (CNRA) de la Mé, qui a bien voulu aider à la mise en œuvre de ce travail.

\section{References:}

1. Avit L.F.J.B., Pedia P.L., Sankaré Y., 1999. Diversité biologique de la Côte d'Ivoire. Rapport de synthèse. Ministère de l'Environnement et de la Forêt. 273 p.

2. Bakayoko S., Abobi A.H.D., Konate Z., Toure N.U., 2019. Effets comparés de la bouse de bovins séchée et de la sciure de bois sur la croissance et le rendement du maïs (Zea mays L.). Agronomie Africaine, $\mathrm{N}^{\circ}$ Spécial (8).

3. Beenaert F., Bitongo D., 2004. Simple end practical methods to evaluate analytical data of soil profiles. Soil science Departement. Belgian Cooperation-Centre Universitaire de Dschang, Cameroun, 66p.

4. Caliman J.P., 2007. La fertilisation du palmier à huile. Performance des systèmes de culture des plantes pérennes, Cirad, Riau, Indonésie, 2p.

5. Cedra C., 1997. Les matériels de fertilisation et de traitement des cultures. Collections Formagri. Bialec (France), 343 p.

6. Cheyns E., Akindes F. et Adié A.F., 2000. "La filière palmier à huile en Côte d'Ivoire 3 ans après la privatisation : état des lieux d'un procès de recomposition institutionnelle, OCL. Oléagineux Corps gras Lipides, 7 (2): 166-171. https://doi.org/10.1051/ocl.2000.0166

7. CNUCED, 2016. Huile de palme. Conférence des nations unies sur le commerce et le développement, New york et Génève, 21 p. 
8. Guilbault P., 2004. Gestion de l'azote à la vigne et au chai. Synthèse d'expérimentation 2000-2003. Contrat de plan Etat-Région Aquitaine. Chambre d'Agriculture de la Gironde, $24 \mathrm{p}$.

9. Jacquemard J.C., 2011. Le palmier à huile. Collection «Agricultures tropicales en poche», Ed. Quæ, Versailles, 275 p.

10. Jacquemard JC. 2013. Le palmier à huile en plantation villageoise. Collection «Agricultures tropicales en poche», Ed. Quæ, Versailles, $142 \mathrm{p}$.

11. Jean-Damien C., 2004. La bouse: historique, importance et écosystème. Thèse pour le Doctorat Vétérinaire : Diplôme d'état, Ecole Nationale Vétérinaire de Toulouse, France, 82 p.

12. Kabrah Y. et Ballo K., 2000. "Caractéristiques chimiques du sol sous palmeraie"Cahiers Agricultures, 9(1), 73-76.

13. Koutou A., CochardB., Durand- gasselin T., 2014. "Fluidité de l'huile produite par des arbres du second cycle de sélection récurrente réciproque chez le palmier à huile (Elaeis guineensis JACQ)", Agronomie Africaine, 26 (3): 275-280.

14. LeCerf J.M., Cagliari S.B., Hornstra G., 2016. L'histoire de l'huile de palme : Faits et chiffres. European Palm Oil Alliance, 18 p.

15. Ognalaga M., M’Akoué D.M., MedzaMve S.D., Ovono P.O., 2017. "Effet de la bouse de vaches, du NPK 151515 et de l'urée à 46\% sur la croissance et la production du manioc (ManihotesculentaCrantz var 0018) au Sud-Est du Gabon (Franceville)", Journal of Animal \&Plant Sciences, 31 (3) 5063-5073. http://www.m.elewa.org/JAPS

16. Palmafrique, 2018. Le palmier à huile dans l'économie Ivoirienne. [Online]

http://www.palmafrique.com/lhuile-de-palme-dans-leconomieivoirienne/(06 Juin 2020).

17. Palmci. 2014. Rapport annuel, 25 p.

18. Rival A., Levang P., 2013. La palme des controverses : Palmier à huile et enjeux de développement, Ed. Quae, 38 - 49.

19. Tano E.K., Konan J.N., Alla-N'Nan O., Akanvou R., Nguetta A.S.P., Konan E.K., 2019."Etude des performances génétiques des descendances parentales issues de deux systèmes de reproduction de géniteurs utilisés en production de semences sélectionnées de palmier à huile (Elaeis guineensisJacq.)", International Journal of Biological and Chemical Sciences,13 (3) : 1800-1816.

20. Tognetti C., Mazzarino M.J., Laos F., 2008. "Compost of municipal organic waste: effects of different management practices on degrability and nutrient release capacity", Soil Biology and Biochemistry, 49: 2290-2296. 
21. Tougma R., 2006. Effets de la fertilisation organique et minérale sur la production en biomasse et en huile essentielle de la citronnelle (Cymbopogoncitratus (D.C) STAPF) dans la région des cascades (Ouest du Burkina Faso). Mémoire de fin d'études de l'IDR, Université Polytechnique de Bobo-Dioulasso (Burkina Faso), 60 p. 